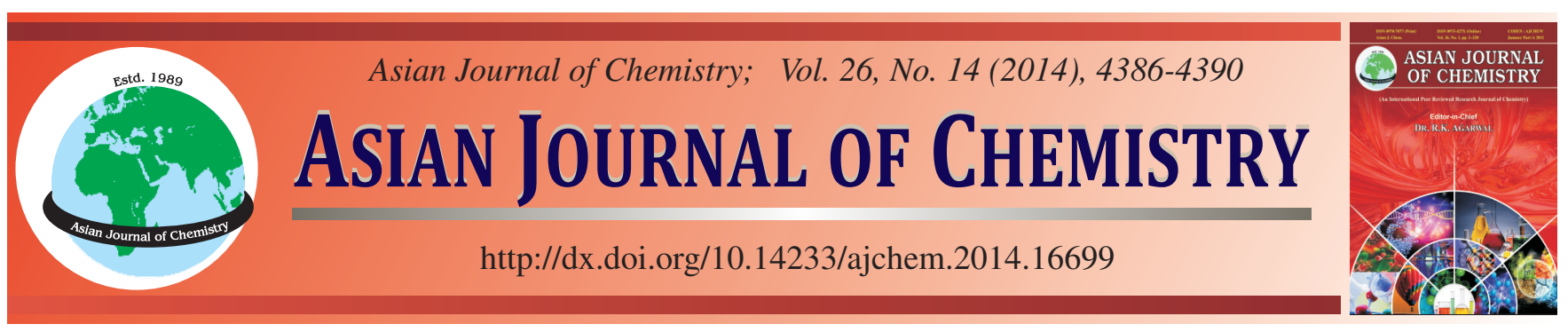

\title{
Comparative Study of Biopolymers Action on Physico-Chemical and Rheological Properties of Water-Based Drilling Muds
}

\author{
Abdelhak Bentriou ${ }^{1}$, Karim Fourar $^{2}$, Abdelbaki Benmounah $^{2}$ and Bahim SAFi ${ }^{2 * *}$
}

${ }^{1}$ Mineral Resource and Energy Laboratory, Faculty of Hydrocarbons and Chemistry, Boumerdes University, Boumerdes, Algeria

${ }^{2}$ Research Unit Materials, Processes and Environment (UR/MPE), Boumerdes University, Boumerdes, Algeria

*Corresponding author: E-mail: safi_b73@umbb.dz

Published online: 5 July 2014;

AJC-15481

\begin{abstract}
Several polymer types compounds are currently used in the water-based mud's, as a filtration control agents, viscosity agent, etc. However, each type of these polymers may have a different effect on the physical, chemical and rheological properties of drilling fluids especially in the presence of an inorganic material such as bentonite. In addition, under geological conditions of wells and during the drilling operation, some compositions of the water-based mud's (clay-polymer system), is sometimes not effective for the operation success of the drilling oil wells in Algeria (South Algeria). For this purpose, the aim of this present work is essentially the study of the polymer actions (xanthan, cellulose polyanionic, partially hydrolyzed polyacrylamide) on the physical, chemical and rheological properties of the drilling muds in the presence of bentonite. An experimental study was conducted on formulations of water-based muds with different types of biopolymers. The results shows that the electrokinetic and rheological study of drilling mud's based different polymers, has permitted to better understanding of the interaction of water-polymers system at different concentrations. Also, the best compositions of drilling mud with concentrations of bipolymers can be used for drilling of wells.
\end{abstract}

Keywords: Biopolymer, Drilling mud, Xanthan, Bentonite, Plastic viscosity, Shear stress, $\zeta$-Potential, Ph.

\section{INTRODUCTION}

The success of drilling of an oil or gas depends mainly on the right choice of drilling fluids used. The optimization of a formulation of a drilling mud can be reduce significantly the overall cost of drilling a well ${ }^{1,2}$. For this purpose, the drilling mud is chosen according to the nature of training, the architecture of the well, the economic objectives and the environmental constraints. In the composition of a water-based drilling mud, the bentonite is not the only element used as viscosifiant. For example, the Xanthan gum (XG) is a natural biopolymers ${ }^{3}$, most common used as a viscosifier in the drilling fluids due to its interesting rheological properties such as viscosity improvement ${ }^{1,4}$. However, polymers are often added in the drilling fluid in order to get adequate properties and permitting to ensure numerous functions and facilitate a good drilling operation process $^{1,2}$.

In fact, the addition of polymers to the drilling fluids provokes an important rheological properties modification. In general, these materials behaviour laws seem complex because of their limited thixotropy, rheofluidifying character and their rigidity which makes them viscoelastic. Several studies were established on the rheological properties of drilling fluids biopolymers. The studies carried have shown that only the type of polymer is different considering its molecular configuration, its rheological behaviour in aqueous medium as well as the viscosifier properties which it confers on drilling mud ${ }^{4}$. The good knowledge of the fluid rheological properties as well as the comprehension of physico-chemical interactions in these charged colloidal systems, are precious elements to adapt the fluid composition to the drilling conditions. Today's literature $^{1,4,5}$ is rich in contributions treating, from one hand, rheological and colloidal properties of clay suspensions (with and without additives) and, on the other hand, the nature of the interactions between their components ${ }^{6,7}$. Nevertheless, the relationship between the rheological and the physico-chemical properties of these suspensions has been well established, mainly for the clay-anionic polymers mixtures often used the drilling fluids formulation ${ }^{6,8,9}$. The previous study shows the effectiveness of some biopolymers as filtration control agent for drilling $\operatorname{mud}^{4,10}$. Moreover, in other studies initiated on drilling muds containing water-based bentonite, the results show that the presence of bentonite can modify the physicochemical and rheological properties ${ }^{11}$. It results the action and the nature of some biopolymers, deserve study. It was also suggested by some authors, to study the stability of the suspensions containing the complicated system (clay-waterpolymer). However, it is difficult to graft two groups using 
this process, especially, when one is hydrophilic and the other is hydrophobic in one reaction because they need different solvents.

For this fact, the main objective of this work is to study the polymers action on the physico-chemical and rheological properties of the water-clay-polymers system in order to bring the elements which contribute to the comprehension of the relation between the measured rheological properties and the structure of these suspensions (water-based mud), particularly on the bentonite-anionic polymers systems.

\section{EXPERIMENTAL}

The bentonite used in this work is a local bentonite. The results of the conformity of bentonite are given in Table-1. From the obtained results, it is noticed that the Maghnia bentonite is conforming to the API 13A norm. The chemical composition of bentonite used is given in Table- 2 .

\begin{tabular}{ccc}
\multicolumn{3}{c}{ TABLE-1 } \\
BENTONITE CHARACTERISATION \\
\hline Parameters & $\begin{array}{c}\text { Requirements of } \\
\text { the standard API }\end{array}$ & Values troves \\
\hline Content of humidity & $10 \%$ max. & $10 \%$ \\
reading in $600 \mathrm{tr} /$ & 22 min. & 29 \\
Yv $(\mathrm{Ib} . / 100 \mathrm{ft} 2$ & $3 * \mathrm{Vp}$ max. & $\mathrm{Vp}=12 \mathrm{Cp}, \mathrm{Yv}=25$ \\
filtrate $(\mathrm{mL}) \mathrm{pH}$ & $15 \mathrm{~mL}$ max. & 11 \\
& 9.5 max. & 9.4 \\
\hline
\end{tabular}

\begin{tabular}{|c|c|c|c|c|c|c|c|c|}
\hline \multicolumn{9}{|c|}{$\begin{array}{c}\text { TABLE-2 } \\
\text { BENTONITE CHEMICAL COMPOSITION }\end{array}$} \\
\hline Ox & $\mathrm{SiO}_{2}$ & $\mathrm{Al}_{2} \mathrm{O}_{3}$ & $\mathrm{Fe}_{2} \mathrm{O}_{3}$ & $\mathrm{Na}_{2} \mathrm{O}$ & $\mathrm{K}_{2} \mathrm{O}$ & $\mathrm{MgO}$ & $\mathrm{MnO}$ & PAF \\
\hline$\%$ & 46.12 & 18.03 & 1.62 & 1.19 & 0.65 & 0.87 & 0.16 & 28 \\
\hline
\end{tabular}

Polymers: The preparation of single polymer and in combination solutions will be carried out under a predefined experimental protocol, when for the complete mud; it is carried out following an operator mode given by the API recommended procedures. The chronological evolution of bentonitic muds drilling fluids technology, with phosphates, tanins/soda or FCL, saturated salted mud, lined derivative system, with silicates introduction, glycols, partially hydrolyzed polyacrylamide, of biopolymers shows the problem complexity in the appropriate additive choice.

The polymers and other products entering in the formulation of different drilling fluid systems will be prepared during this work are commercial products having the mentioned characteristics on the technical sheets presented by the manufacturer.

Xanthan gum (XCD): The xanthene rubber is a biodegradable polymer of a high molecular weight semi-rigid, from the polysaccharides group. It is presented under the form of yellow powder and used, even with small quantities, to increase the viscosity and the suspension power of the water based systems. The xanthene rubber also possesses the capacity to produce a rheofluidifiant system and develop a real gel structure. Its average molecular formula is approximate

$$
\left(\mathrm{C}_{67} \mathrm{H}_{102} \mathrm{O}_{56}\right)_{\mathrm{n}}, \mathrm{n}=830 \text { in } 2800
$$

Cellulose polyanionic: The cellulose polyanionic is a cellulosic polymer derived from CMC (cellulose carboxy- methyl) which reduces the filtrate and plays the role of viscosifiant in the water based drilling fluids formulation. One of the cellulose polyanionic characteristics is the substitution degree (DS) of the carboxymethyl group which is higher than 0,8 . The cellulose polyanionic is known to have a thermal stability limit around $150{ }^{\circ} \mathrm{C}$, which is higher to the $\operatorname{starch}^{12}$.

Partially hydrolyzed polyacrylamide: The partially hydrolyzed polyacrylamide, is a high molecular weight polymer, which is under a liquid form, and an opaque appearance and cream like colour. An anionic destined to bring an encapsulation and a stabilisation of opaque clays. The partially hydrolyzed polyacrylamid also plays a viscosifiant, friction reducer and flocculent role. It is also a filtrate reducer which can be used in a wide range of systems with low or high solid content with soft or salted water.

Conformity test of bentonite: This test is carried out following API 13A SEC 4 norme. A $22.5 \mathrm{~g}$ dissolved bentonite suspension in $350 \mathrm{~mL}$ of distilled water is aged during $24 \mathrm{~h}$ at $\mathrm{T}=16^{\circ} \mathrm{C}$. The required trials are the filtrate volume (during 30 min under a pressure of 7 bars), the plastic viscosity (VP), the yield value (Yp) and the $\mathrm{pH}$.

Electrokinetic potential test: The effect of different biopolymers concentrations on the zeta potential of drilling muds studied was determined on a Malvern Instruments ZETASIZER 2000 particle seizer. The measuring principle is based on the electrophoretic mobility of charged particles in suspension. Under the effect of the electric field applied a displacement of the charged particles to the oppositely charged electrode (Fig. 1). After the bombardments of the particles (studied the suspension), the refracted laser will be collected using a correlator in order to transform announces it in data of measurement of potential of the electric double-layer of the particle. One $\mathrm{cm}^{3}$ of the studied suspension was diluted in $30 \mathrm{~cm}^{3}$ of distilled water, after which $5 \mathrm{~mL}$ of this suspension was injected into the analyzer.

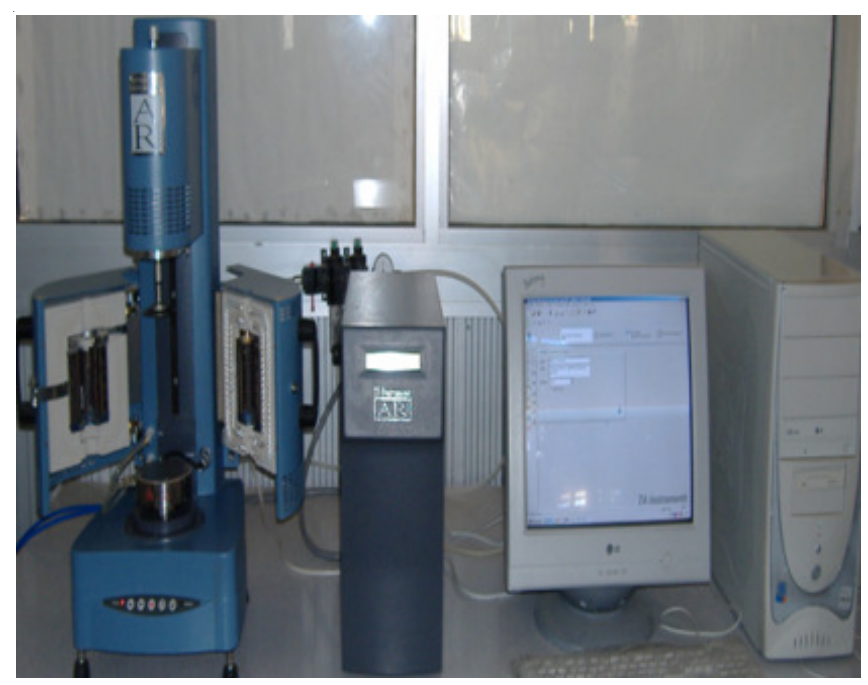

Fig. 1. Rheometer AR 2000.-TA

Rheological test: All rheological measurements were conducted on a TA Instruments AR2000 rotary rheometer (Fig. 2) with imposed constraint and a controlled speed able to test different sample types. The system used in this study is 


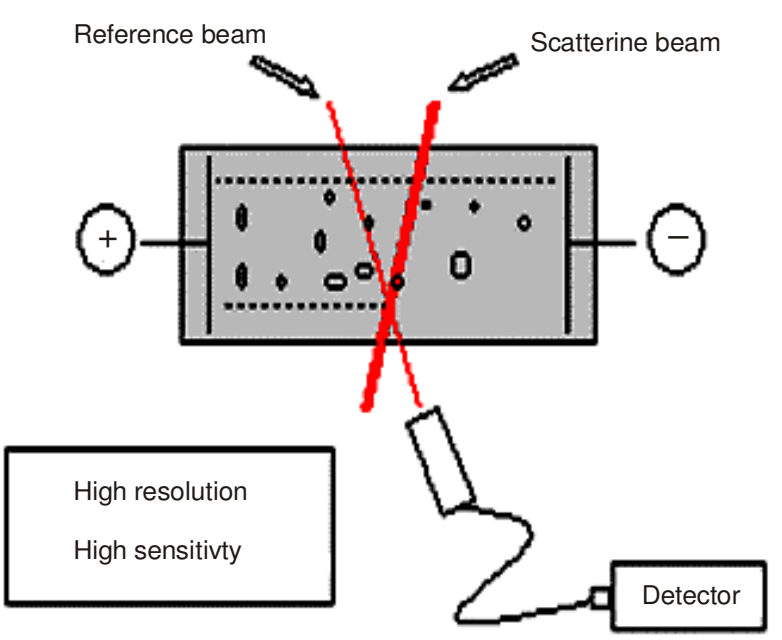

Fig. 2. Measuring principle of $\zeta$-potential (ZETASIZER MALVERN)

the cone-plate for the polymer solutions and polymer mud, where the sample is placed between a cone $60 \mathrm{~mm}$ radius and $2^{\circ}$ an angle and a planar surface perpendicular to the axis the peltier cone. The diameter and angle choice of the cone are depending on the experimental conditions and the sample viscosity. A thermal control system was used to maintain a constant temperature.

\section{RESULTS AND DISCUSSION}

Xanthan solution: The rheological properties of xanthan solutions depend first of the molecules association degree and then of the polymer concentration regime. It means the molecular mass is an important parameter that largely influences the rheological results.

Once dispersed in water, the xanthan molecules which have a high weight molecular, form complex aggregates formed by hydrogen bonding and entanglement of the polymer chains. Because of these highly organized networks, xanthan solutions show a high viscosity with a low shear rates but at larger shear rate a decrease the viscosity of the suspension was observed. This may be due to the disintegration of the network and the alignment of different macromolecules in the direction of shear.

According to the rheograms (Fig. 3) all the solutions have a non Newtonian flow (non Newtonian behaviuor). This finding was confirmed by several authors ${ }^{13,14}$. The viscosity variation of xanthenes aqueous dispersions, at different concentrations, has been examined in function of the shear gradient: the viscosity decreases with the shear ratio (decreasing shear) ${ }^{14}$. However, the xanthenes viscosity has increased with its concentration. An important xanthenes theology characteristic is the presence of a limit constraint, which represents the required effort to start the flow ${ }^{14,15}$. This constraint-decreases with the concentration decrease. This is probably due to a formation of a gel with a weak structure, due to the insufficient polymer quantity in the solution.

The Herschel Bulkely modal is the one that suits the best to describe the solutions rheological properties. The xanthenes rubber behaved in solution as a pseudo plastic fluid with a limit constraint ${ }^{7}$. From Fig. 4, the $\zeta$-potential increase in function of the concentration can be explained by the load contribution. The polymer chains density loads with a negative sign increases

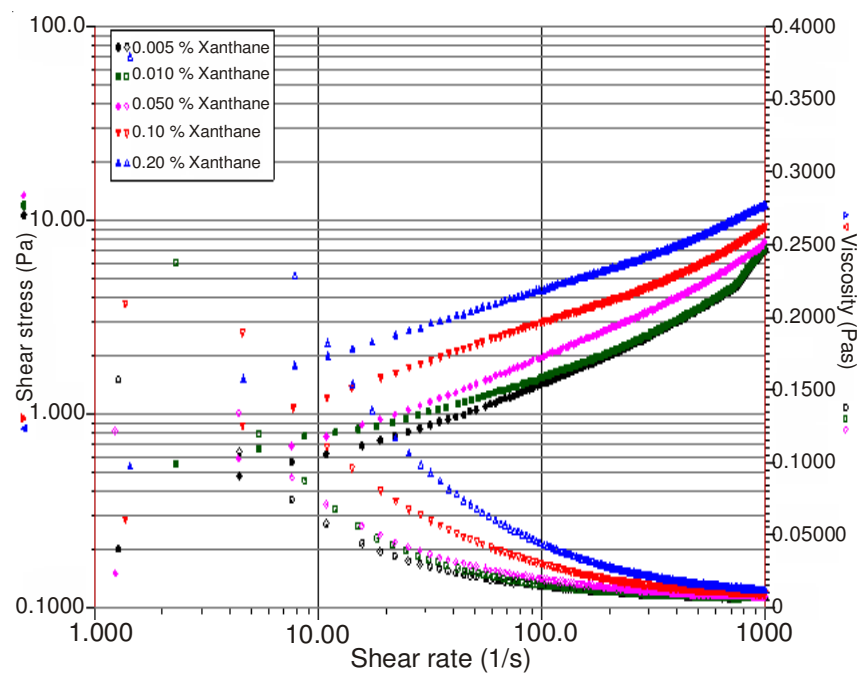

Fig. 3. Rheogrammes of xanthan solutions

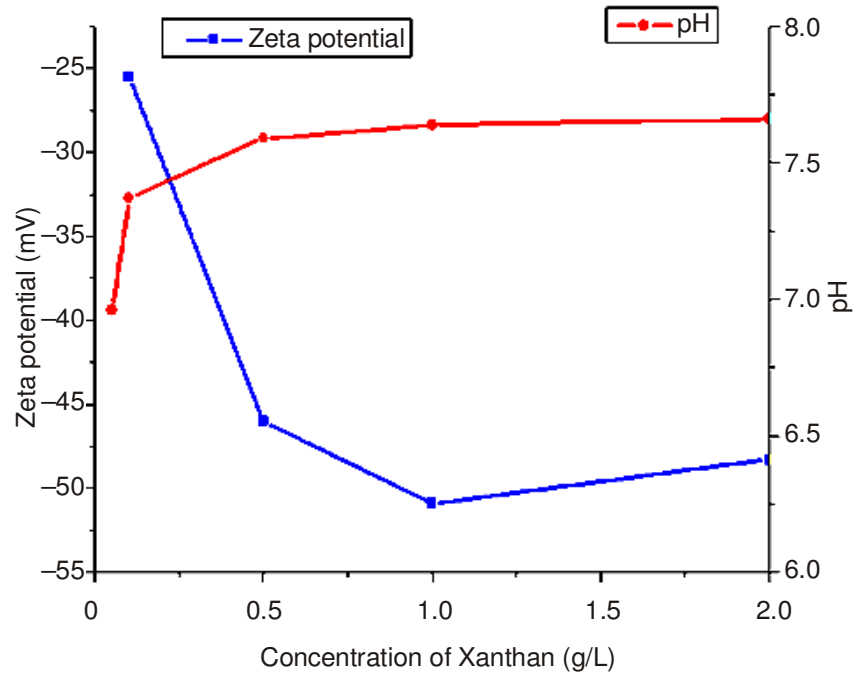

Fig. 4. Xanthan concentration effect on the zeta and the $\mathrm{pH}$ potential

in the medium, which explains this $\zeta$-potential increase, then it slightly decreases for a $2 \mathrm{~g} / \mathrm{L}$ concentration certainly linked to the medium load saturation. The $\mathrm{pH}$ increases then stabilize for a value $\approx 6$.

Fig. 5 reveals that with the xanthenes rubber concentration increase, the viscosity increases, with is normal. This increase is reverberated on the transmission which decreases (from 94, 3 to $62,3 \%$ ) and increases in function of the xanthenes solutions ageing time.

Cellulose polyanionic solutions: Same as for the xanthenes solutions, Fig. 6 the cellulose polyanionic solutions flow curves show a pseudo plastic (rheofluidifiant) character obeying to the power law rheological model, where the viscosity increases with the increase of the cellulose polyanionic concentration and decreases with the shear ratio increases.

The cellulose polyanionic is a cellulosic polymer of carboxymethylcellulose type having a flexible conformation ball, contrarily to the xanthenes rubber which is a polysaccharide appearing in the form of semi-rigid impeller. Fig. 7 shows the viscosity increases with the cellulose polyanionic concentration increase. This behaviour is explained by the fact that the carboxyl group in the cellulose polyanionic molecule 


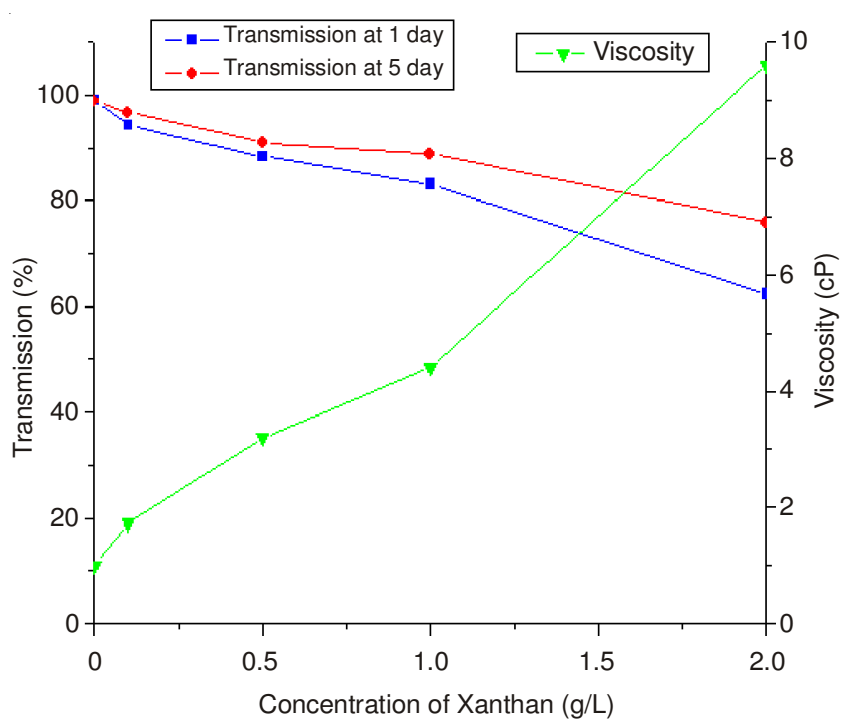

Fig. 5. Xanthan concentration effect on the transmission and viscosity

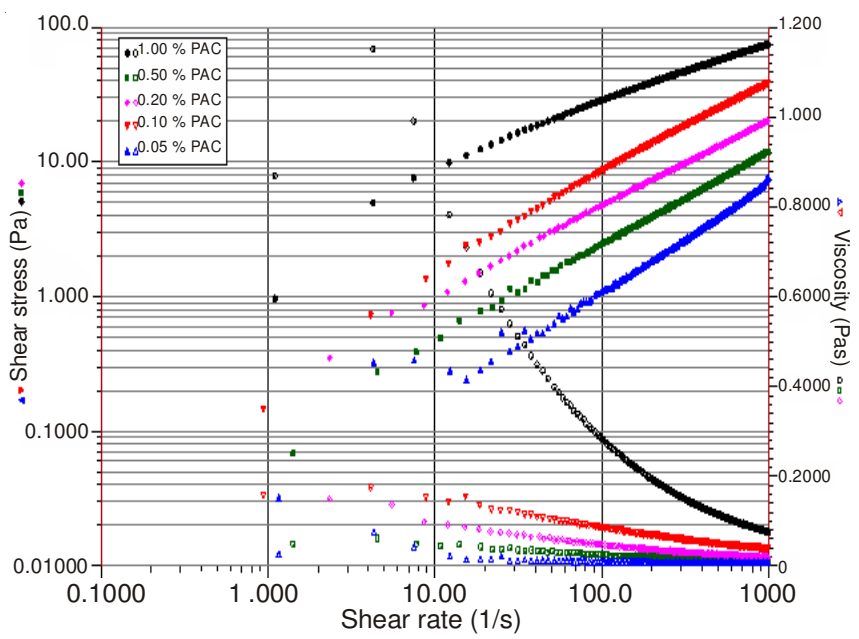

Fig. 6. Cellulose polyanionic solutions rheogrammes

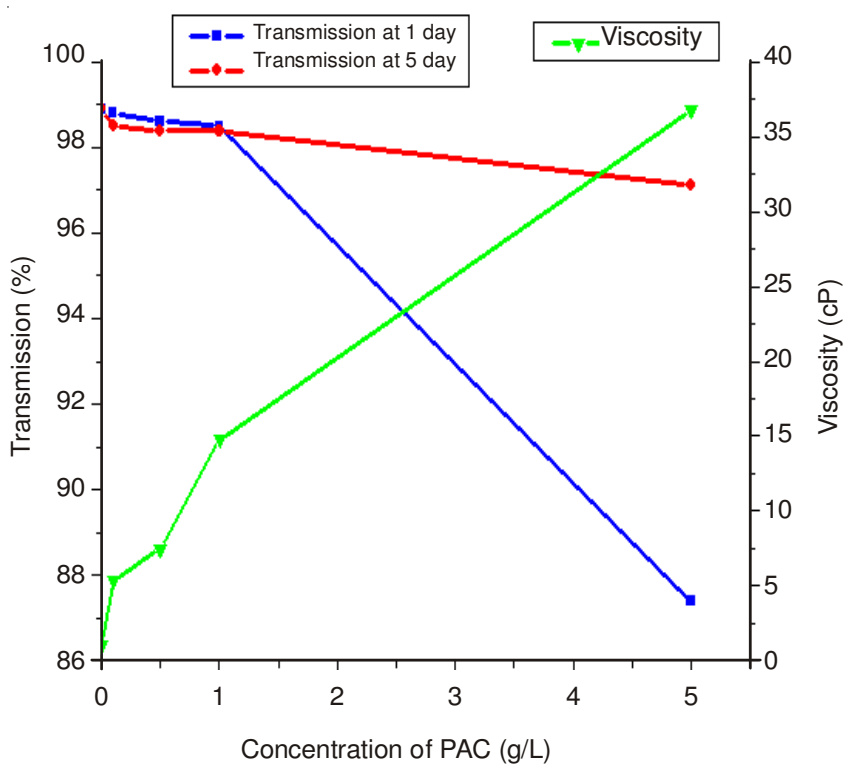

Fig. 7. Cellulose polyanionic concentration effect on the transmission and viscosity favours a good dispersion in water, leading to friction increase between particles ${ }^{12}$. The cellulose polyanionic $\mathrm{Na}^{+}$ion dissociation creates negative sites along the polymer chain. A mutual repulsion between charges that the chains will be randomly coiled and extend linearly, causing an increase in viscosity.

Fig. 8 shows $\zeta$-potential increase until a value of $-65,3$ $\mathrm{mV}$. It is noted that the cellulose polyanionic gives the highest potential values compared to other polymers. The $\mathrm{pH}$ of cellulose polyanionic solutions increases with the introduction of the latter in water and stabilize at values near 7.7.

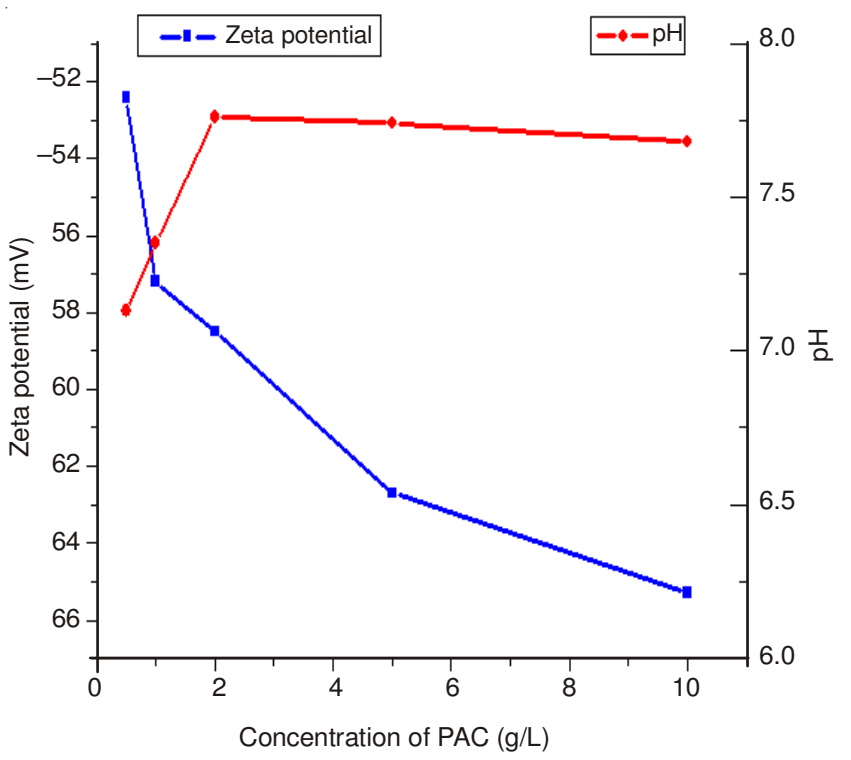

Fig. 8. Cellulose polyanionic concentration effect on the zeta potential and $\mathrm{pH}$

The cellulose polyanionic solutions transmission decreases sharply with the polyanionic cellulose concentration increase. This is due to non total dissolution of polymer solid grains, then it increases becomes stable at $5 \mathrm{~g} / \mathrm{L}$ after 5 days.

Solutions de partially hydrolyzed polyacrylamide: Fig. 9 illustrates the partially hydrolyzed polyacrylamide solutions rheological behaviour. For xanthenes and cellulose polyanionic solutions, an increase in the viscosity is observed with a concentration increase. This is valid for the xanthenes and cellulose polyanionic solutions, the systems rheofluidifiant character is clearly indicated on Fig. 10 where the viscosity decreases with the shear ratio. The partially hydrolyzed polyacrylamide solutions zeta potential load increases to stabilise near $-44 \mathrm{mV}$ and the $\mathrm{pH}$ decreases when adding partially hydrolyzed polyacrylamide and stabilize at values around $7.4^{16,17}$ (Fig. 11).

\section{Conclusions}

- The drilling fluids formulation as well as the new technology related to the used tools has known a great evolution in order to bring the best answers to the encountered problems and ensure a continuous amelioration of drilling operations. Several works have shown, during the drilling, the open whole walls stability is closely linked to several phenomena including fluids-rock interactions, rock mechanical aspect and the abnormal behaviour of the crossed geological rock layers as well as the certain inappropriate drilling practices. 


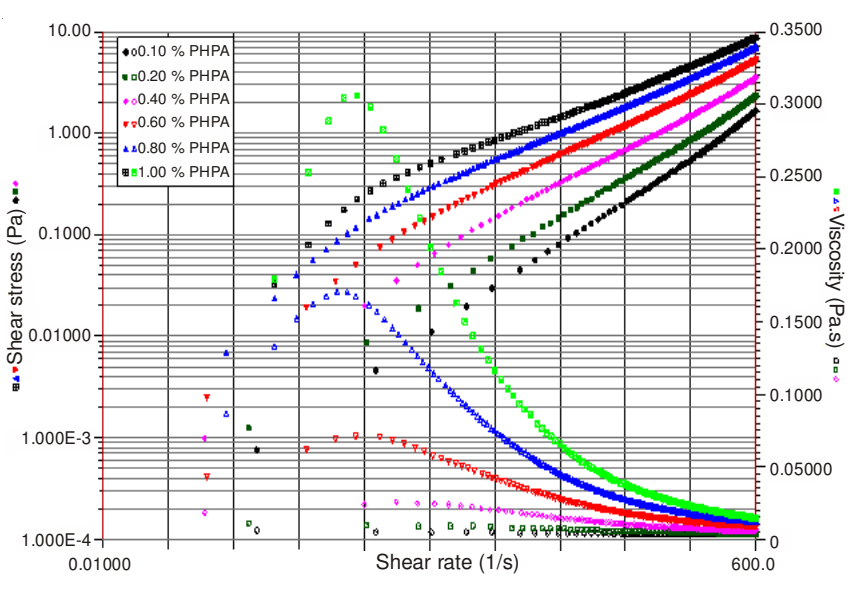

Fig. 9. Partially hydrolysed polyacrylamid solutions rheogrammes

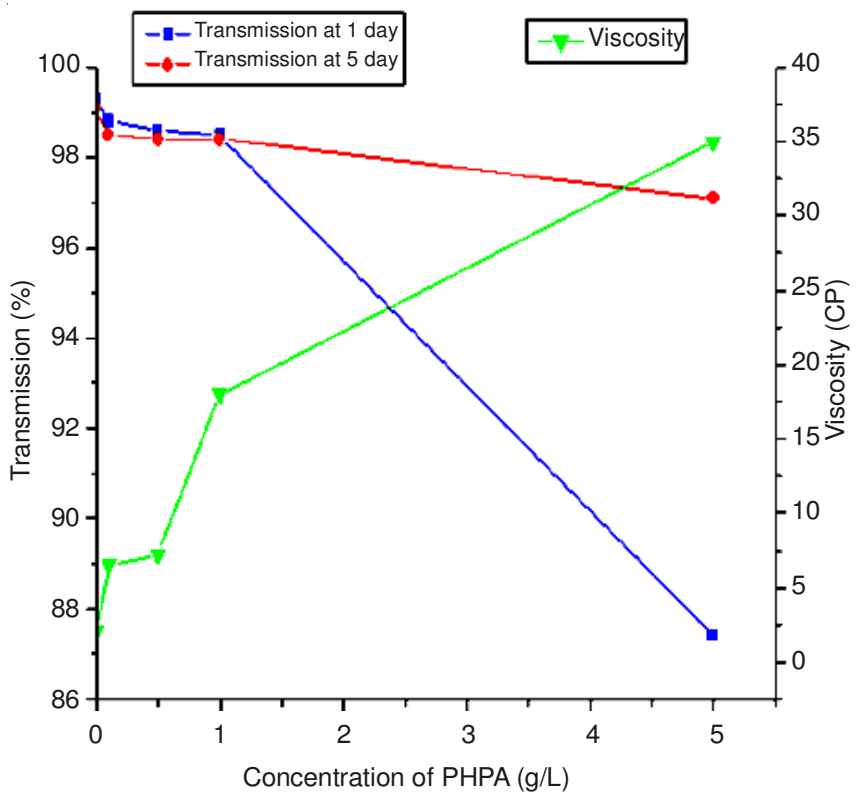

Fig. 10. Partially hydrolysed polyacrylamid concentration effect on the transmission and viscosity

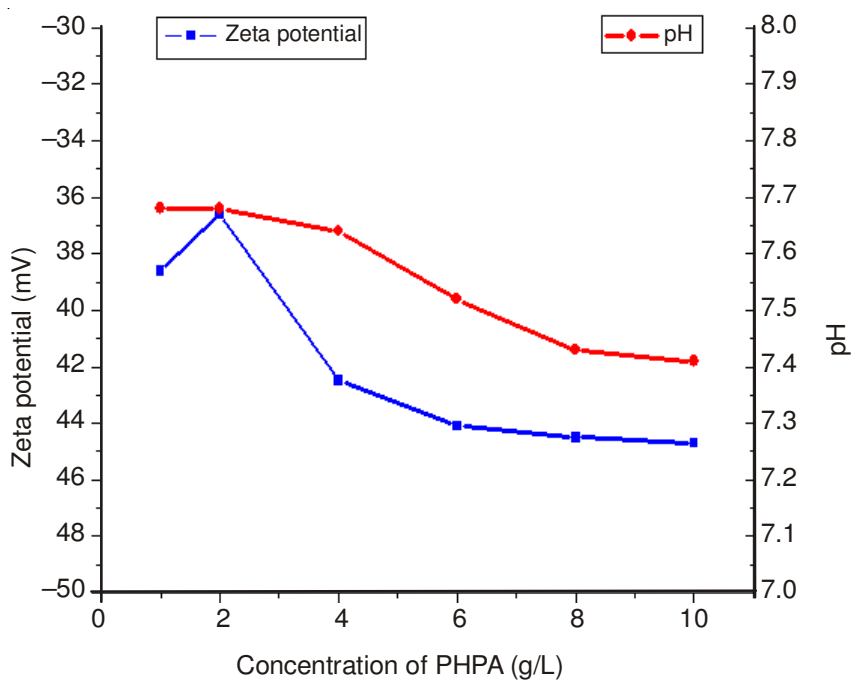

Fig. 11. Partially hydrolysed polyacrylamid concentration effect on the $\zeta$-potential and $\mathrm{pH}$
- The rheological and electrokinetic study of polymer solutions, has permitted to better understand the water-polymers system at different concentrations, of $0,005 \%$ at $0,2 \%$ for the case of xanthenes rubber, of $0,05 \%$ at $1 \%$ for the cellulose polyanionic and of $0,1 \%$ at $1 \%$ for the partially hydrolyzed polyacrylamide.

- The rheofluidifiant behaviour of polymer solutions has been put in evidence in order to take into account these suspensions structural evolution. The Herschel Bulkley rheological law was shown as being the most adequate to describe the rheological behaviour of this type of polymers.

\section{REFERENCES}

1. American Petroleum Institute. Principles of Drilling Fluid Control, University of Texas, Continuing Education Petroleum Extension Service, Austin, edn 12 (1969).

2. C. Garcia and P. Parigo, Drilling Fluids, French Petroleum Institute Technip Edition, Paris (1968).

3. N.J. Alderman, D.R. Babu, T.L. Hughes and G.C. Maitland, Rheological Properties of Water-Based Drilling Muds, 4th International Congress on Rheological, Sydney (1988).

4. S. Baba Hamed and M. Belhadri, J. Petroleum Sci. Eng., 67, 84 (2009).

5. G.R. Gray, H.C. Darely and W.F. Rogers, Composition and Properties of Oil Well Drilling Fluids, Gulf Publishing: Houston, TX, edn 4, pp. 1-36 (1980).

6. M. Khodja, J.P. Canselier, F. Bergaya, K. Fourar, M. Khodja, N. Cohaut and A. Benmounah, Appl. Clay Sci., 49, 383 (2010).

7. R. Schlemmer, J.E. Friedheim, F.B. Growcock, J.A. Headley, S.C. Polnaszek and J.B. Bloys, Membrane Efficiency in Shale-An Empirical Evaluation of Drilling Fluid Chemistries and Implications for Fluid Design. SPE 74557. IADC/SPE Drilling Conference, Dallas (TX), 26-28 February 2002.

8. L.V. Amorim, C.M. Gomes, H.L. Lira, K.B. França and H.C. Ferreira, Mater. Res., 7, 583 (2004).

9. M.V. Kok and T. Alikaya, Energy Sources, 27, 405 (2005).

10. R.M. Pérez, S. Siquier, N. Ramírez, A.J. Müller and A.E. Sáez, J. Petrol. Sci. Eng., 44, 317 (2004).

11. R. Caenn and G.V. Chillingar, J. Petrol. Sci. Eng., 14, 221 (1996).

12. A. Senthil Kumar, V. Mahto and V.P Sharma, Indian J. Chem. Technol., 10, 525 (2003)

13. A. Kayacier and M. Dogan, J. Food Eng., 72, 261 (2006).

14. K. Benyounes and A. Benmounah, Int. J. Phys. Sci., 7, 1790 (2012).

15. J. Ahmed and H.S. Ramaswamy, Food Hydrocoll., 18, 367 (2004).

16. M. Maallem, M. Safi, M. Saidi, A. Benmounah and D. Aboutaleb, Asian J. Chem., 25, 8848 (2013).

17. J.N. Israelachvili and R.M. Pashley, J. Colloid. Interf. Sci., 98, 500 (1984). 